This is the final peer-reviewed accepted manuscript of:

Directly Observing Micelle Fusion and Growth in Solution by Liquid Cell Transmission Electron Microscopy, Lucas R. Parent; Evangelos Bakalis; 5 Abelardo Ramírez-Hernández; Jacquelin K. Kammeyer; Chiwoo Park; Juan de Pablo; Francesco Zerbetto; Joseph P. Patterson; Nathan C. Gianneschi, J. Am. Chem. Soc. 2017, 139, 47, 17140-17151.

The final published version is available online at: https://doi.org/10.1021/jacs.7b09060

Rights / License:

The terms and conditions for the reuse of this version of the manuscript are specified in the publishing policy. For all terms of use and more information see the publisher's website.

This item was downloaded from IRIS Università di Bologna (https://cris.unibo.it/)

When citing, please refer to the published version. 


\title{
Directly Observing Micelle Fusion and Growth in Solution by Liquid Cell Transmission Electron Microscopy
}

\author{
Lucas R. Parent, ${ }^{1,2,3,4}$ Evangelos Bakalis, ${ }^{5}$ Abelardo Ramírez-Hernández, ${ }^{6,7}$ Jacquelin K. Kammeyer, ${ }^{1}$ \\ Chiwoo Park, ${ }^{8}$ Juan de Pablo, ${ }^{6,7}$ Francesco Zerbetto, ${ }^{5}$ Joseph P. Patterson, ${ }^{1,9}$ Nathan C. Gianneschi ${ }^{1,2,3,4^{*}}$ \\ ${ }^{1}$ Department of Chemistry \& Biochemistry, University of California, San Diego, La Jolla, CA 92093. ${ }^{2}$ Department of Chem- \\ istry, Northwestern University, Evanston, IL 60208. ${ }^{3}$ Department of Materials Science \& Engineering, Northwestern Univer- \\ sity, Evanston, IL 60208. ${ }^{4}$ Department of Biomedical Engineering, Evanston, IL 60208. ${ }^{5}$ Dipartimento di Chimica "G. \\ Ciamician", Università di Bologna, Bologna Italy. ${ }^{6}$ Materials Science Division, Argonne National Laboratory, Lemont, IL \\ 60439. ${ }^{7}$ Institute for Molecular Engineering, The University of Chicago, Chicago, IL 60637. ${ }^{8}$ Department of Industrial and \\ Manufacturing Engineering, Florida State University, Tallahassee, FL 32306. ${ }^{9}$ Labratory of Materials and Interface Chemis- \\ try and Center of Multiscale Electron Microscopy, Department of Chemical Engineering and Chemistry, Eindhoven Univer- \\ sity of Technology, Eindhoven, The Netherlands.
}

KEYWORDS: liquid cell (LC)TEM, polymeric micelles, micelle fusion, amphiphilic assemblies.

\begin{abstract}
Amphiphilic small molecules and polymers form commonplace nanoscale macromolecular compartments and bilayers, and as such are truly essential components in all cells and in many cellular processes. The nature of these architectures, including their formation, phase changes, and stimuli-response behaviors are necessary for the most basic functions of life, and over the past half-century, these natural micellar structures have inspired a vast diversity of industrial products, from biomedicines to detergents, lubricants and coatings. The importance of these materials and their ubiquity, have made them the subject of intense investigation regarding their nanoscale dynamics with increasing interest in obtaining sufficient temporal and spatial resolution to directly observe nanoscale processes. However, the vast majority of experimental methods involve either bulk-averaging techniques including light, neutron and X-ray scattering, or are static in nature including even the most advanced cryogenic transmission electron microscopy techniques. Here, we employ in situ liquid cell transmission electron microscopy (LCTEM) to directly observe the evolution of individual amphiphilic block copolymer micellar nanoparticles in solution, in real time with nanometer spatial resolution. These observations, made on a proof-of-concept bioconjugate polymer amphiphile, revealed growth and evolution occurring by unimer addition processes and by particle-particle collision-and-fusion events. The experimental approach, combining direct LCTEM observation, quantitative analysis of LCTEM data, and correlated in silico simulations, provides a unique view of solvated soft matter nano-assemblies as they morph and evolve in time and space, enabling us to capture these phenomena in solution.
\end{abstract}

\section{INTRODUCTION}

The assembly and evolution of soft matter in solution is fundamental to both the formation and functional properties of complex biological and synthetic materials. ${ }^{1}$ There are multiple mechanisms by which particles can undergo phase transitions and growth with the problem of characterizing a given system often approached using powerful, routine, but indirect in situ scattering techniques. ${ }^{2-8}$ These methods provide an adequate bulk-averaged overview of the sample size and morphology based on assumptions of the nature of the nanostructures and theoretical fitting of the raw data. For many systems, including amphiphilic block copolymer (BCP) micelles, a delicate interplay exists between the kinetic and thermodynamic parameters, ${ }^{9-18}$ which govern their size, morphology and dynamic behavior. ${ }^{19-21}$ Ex situ transmission electron microscopy is a powerful tool for directly probing more complex morphologies, allowing characterization on a particle-byparticle basis, complementing bulk solution methods. ${ }^{6,11,12,22-24}$ However, traditional microscopy techniques inherently lack the ability to probe dynamic processes, such as diffusion, morphological transformations or phase changes as they occur on the nanoscale. Consequently, highly complex structures can be prepared by generally predictable, and controllable methods for a wide variety of surfactant or polymer amphiphiles. ${ }^{25-27}$ However, we lack techniques for directly observing nanoparticle-by-nanoparticle dynamics and transformations as they occur. $5,7,8,22,28-33$

Typically, amphiphilic BCP assembly is performed via direct dissolution into a selective solvent, ${ }^{2,24}$ or via gradual solvent exchange methods from common solvents to a solvent selective for one blocks. ${ }^{11}$ After solvent-switch, and dependent on the preparation conditions, the assemblies will either equilibrate over time, forming the lowest free energy structure, or be kinetically trapped, yielding metastable morphologies and often resulting in the "freezing" of complex, and functionally desirable morphologies. ${ }^{9,24,34,35}$ For kinetically trapped assemblies, morphological reorganization and equilibration can occur following physical or chemical perturbation to the system. ${ }^{5,67}$ Epps et al. recently reported BCP micelle sizeevolution that was physically-induced and likely interfacedriven, providing experimental evidence that small, kinetically frozen micelles can undergo dynamic transformations that are 
highly suggestive of particle-particle fusion events. ${ }^{30,31}$ However, these types of dynamic nanoscale processes in micelle assemblies have never been directly observed, and the postulated underlying mechanisms, such as unimer insertion, fusion, fission, complete micelle disassembly and reassembly, or nucleation-elongation are the subject of investigation in many specific systems and classes of polymer and small molecule surfactant assemblies. ${ }^{3,7-9,35-45}$ That is, for responsive materials that undergo phase transitions, or materials that spontaneously undergo these processes, there is naturally great interest in precisely how they occur. We demonstrate herein, that liquid cell transmission electron microscopy (LCTEM) is a feasible and powerful method for observing soft matter phase transitions that should be added to the suite of characterization methods for nanomaterials in general. We show this for a proof-of-principle case, utilizing a biohybrid polymer system. $^{46-51}$

Here, we employ LCTEM videography (Videos S1-S11), to visually capture morphology switching and growth processes of BCP micelles. The assemblies evolve from small spherical micelles into larger bicontinuous morphologies while being observed directly on a particle-by-particle basis. Growth occurs through multiple simultaneous pathways that exist over all micelle size regimes, namely by micelle-micelle fusion $^{7,8,36,37}$ and by unimer addition. ${ }^{3,9,41,52}$ By monitoring the motion and evolution of individual micelles in real time with nanometer spatial resolution, we were able to quantify the diffusion behavior of individual assemblies in solution, and directly observe fusion events and micelle-micelle interactions that do not result in fusion. The experiments conducted here involve four key parts that we aim to be generally instructive in terms of utilizing this technique for soft matter in motion: 1) Setting up the LCTEM experiment and collecting direct video data; 2) Detailed video analysis of individual micelle motion by combining an automated object-tracking algorithm, with a detailed examination of the complexities of anomalous diffusive motion; 3) Quantitative analysis of morphologies, sizes, and interactions over time (collisions, fusion events, individual particle growth in the absence of interactions with other particles); and 4) Molecular modeling and dynamical simulation of interactions/transitions observed.

\section{RESULTS AND DISCUSSION}

Micelle Assembly and Morphology Transition in Bulk Solution. A block copolymer amphiphile (phenyl- $b$-peptideco-hydroxyl) was prepared via ring-opening metathesis polymerization using the Grubbs initiator ( $\mathrm{H}_{2} \mathrm{IMES}$ )$(\text { pyr })_{2}(\mathrm{Cl})_{2} \mathrm{Ru}=\mathrm{CHPh}$ (see Methods and Figure S1). ${ }^{51}$ Spherical micelles were formed from the amphiphilic BCP initially dissolved in dimethylformamide and then switched to water by slow addition, followed by a final change to Dulbecco's phosphate-buffered saline (DPBS) solution by dialysis yielding a pure aqueous formulation (polymer structure depicted in Figure $1 \mathrm{~A}$ and Figure S1). These micelles were characterized by cryo-TEM (Figure 1A Figure S2) and dynamic light scattering, DLS (Figure S3), both confirming the formation of micelles, predominantly of $\sim 20 \mathrm{~nm}$ diameters, which are stable over several months at quiescent conditions. Due to the relative hydrophobic/hydrophilic block lengths, the corona layer is thin in the spherical micelles, on the scale of $\sim 2-4 \mathrm{~nm}$. Physical perturbation of the initial micelle solution, by vortexing or by controlled evaporation, resulted in their rapid (within several minutes to hours) reorganization into larger assemblies (Figure 1B,C) with complex morphologies and internalized water pockets, ${ }^{7,9-11,53-56}$ which were found in abundance by cryo-TEM characterization (Figure 1D-G and Figure S2). After such perturbation and the observed size/morphology transition, the system did not relax back to the original predominant morphology, indicating that the initial small, spherical micelles were kinetically trapped structures.

In situ LCTEM Imaging/Video and Analysis of Micelle Dynamic Motion. In situ LCTEM imaging experiments (Figure 2) were performed by loading a sub- $\mu \mathrm{L}$ volume of the initial, as-formed spherical micelle solution between two glow-discharged $\mathrm{SiN}_{\mathrm{x}}$ membrane windows in a commercial liquid flow TEM holder (details in Methods), to form a sealed "liquid-cell" (Figure 2A). With the liquid-cell inside the TEM, in situ LCTEM videographic observations were performed at five different dose rates $\left(1.6 \mathrm{e}^{-} / \AA^{2} \mathrm{~s}, 2.7 \mathrm{e}^{-} / \AA^{2} \mathrm{~s}, 6 \mathrm{e}^{-} / \AA^{2} \mathrm{~s}, 8.4 \mathrm{e}^{-}\right.$ $/ \AA^{2} \mathrm{~s}$, and $13.4 \mathrm{e}^{-} / \AA^{2} \mathrm{~s}$, Table S1), safely below the threshold for irreversible degradation of buffered-water. ${ }^{57}$ Minimizing electron dose during LCTEM experiments is crucial, especially for
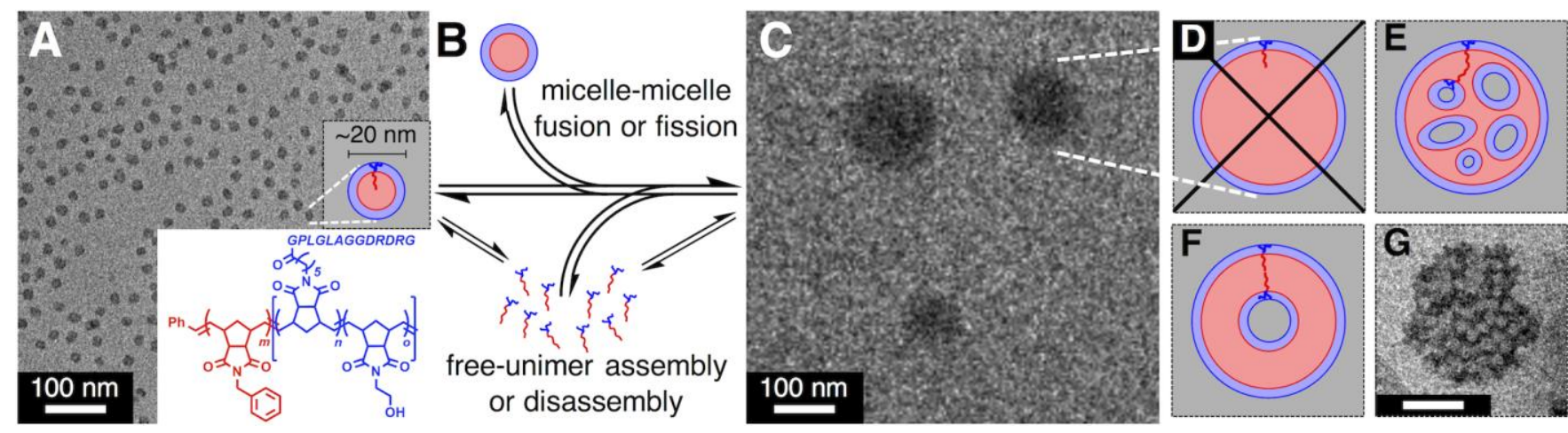

Figure 1. Evolution processes of kinetically trapped micelles in solution. (A) Cryo-TEM image of the initial, kinetically trapped phenyl- $b$ peptide-co-hydroxyl micelles ( $20 \mathrm{~nm}$ diam.). Insert shows the polymer structure. (B) Potential size/morphology evolution processes, micelle-micelle fusion/fission or unimer assembly/disassembly. (C) LCTEM image of the larger micelles observed in the liquid-cell. (D,E,F) Possible internal structures of the larger LCTEM micelles; (D) large crew-cut spherical micelle (not possible to form for this BCP unimer), (E) bicontinuous micelle, and (F) vesicular micelle (polymersome). (G) Cryo-TEM image of the initial solid-sphere micelles after bulk solution perturbation by controlled solution evaporation (larger assemblies with distinct bicontinuous morphologies and external-surface diameters similar to the large micelles observed in LCTEM). Scale bar is $100 \mathrm{~nm}$. 
experiments involving soft matter nanostructures held together largely through secondary forces, as beam-related artifacts at high dose rates can influence individual object motion and overall system equilibrium and kinetics, altering solution chemistry through radiolysis and creating reactive species. ${ }^{57-62}$ We note that no bubble formation or sample degradation were observed during these experiments, and at the dose rates used, the theoretical temperature change is negligible, giving no significant beam-induced heating. ${ }^{62}$

At the very start of the LCTEM observation (i.e. the very first $\mathrm{e}^{-}$beam irradiation of the sample, and $\sim 8$ min after assembling the liquid-cell), a distribution of mobile micelles was observed with diameters ranging from $\sim 40$ to $160 \mathrm{~nm}$ (Video S1, Table S2, Figure S4). Due to the very large windowsolution interface within the liquid-cell once assembled, and the manual process of assembly, we infer that the small kinetically-trapped spherical micelles had been perturbed to evolve into these larger morphologies, similar to the size and morphology transitions we observed in bulk solution following perturbation (Figure 1 and Figure S2). We note that for the length of the BCP chain used ( $\sim 15 \mathrm{~nm}$ fully extended) to form assemblies of this size (>40 nm diameter) some internal volume must be occupied by water (Figure 1C-F). As we continued to monitor the motion and evolution of these micelles in the liquid-cell, complex interactions were captured (collisions, fusion events, and non-collision growth, discussed below) in addition to diffusive motion. We first aimed to determine the true nature of micelle motion during LCTEM observations. Here, we were chiefly concerned with understanding to what degree the observed motion was influence by the LCTEM experimental conditions rather than being a manifestation of the intrinsic physical properties of the micelles-solution system alone. This elementary knowledge is necessary to begin to interpret the more complex processes and their driving forces, which are captured in the LCTEM videos, such as fusion events and non-fusion collisions, and individual growth.

The question that naturally arises is whether the dynamics of each micelle is affected directly by the TEM beam, potentially in the form of micelle degradation by radiolysis, $\mathrm{e}^{-}$bombardment momentum transfer, thermal or solution-chemistry gradients. In addition, the particles could be affected indirectly via secondary effects, where the beam changes the local environment, either at the window surfaces or in the solution, which in turn alters micelle motion behavior. Alternatively, motion could be ideally free from $\mathrm{e}^{-}$beam influence, where motion is purely a reflection of the physically properties of the micelles and their 'natural' interactions within the nanoscale confines of the liquid. Using the multi-object tracking algorithm (MOTA) analysis (Table S2,S3,S4), ${ }^{63,64}$ each individual micelle was tracked and measured to determine $\mathrm{x}, \mathrm{y}$ trajectory (Figure 2B,C), cross sectional area, and minor/major-axis diameters for each frame in the LCTEM videos (Supporting Information). We applied anomalous diffusion object motion analysis, ADOMA (Table S5,S6, Figure S5, Supporting Information), to the $\mathrm{x}, \mathrm{y}$-location data to quantify micelle motion, and to understand the stochastic mechanisms underlying their diffusive behavior. Overall, the motion of all individual micelles in these LCTEM experiments is very similar, classified as anomalous sub-diffusion, reflecting fractional Brownian motion (fBm) (Figure 2D and Table S5), and characteristic confined environments, such as the liquid-cell. Motion is a combination of sticking, slipping, and sliding, with the frequency of surface trapping and escaping increasing with dose rate, but with the hopping length remaining constant over all doses (Figure S5). The lack of motion directionality in the micelle trajectories strongly suggests that direct interactions between the $\mathrm{e}^{-}$beam radiation field and the micelles are not significant, but as discussed below, indirect effects are operative. A detailed methods discussion of ADOMA can be found in the Supporting Information.

Applying the method of generalized moments, solving for the structure function (Table S6), finds a multi-fractal nature in the stochastic mechanisms of motion for all 56 micelles observed and tracked by LCTEM. This is generated by the interplay between fractional Gaussian noise (fGn) and Truncated Levy Walks. Similar general structure functions have been identified as the operative mechanisms that drive the motion of a number of experimental and theoretical studies in living cells and lipids, ${ }^{65-69}$ and reflect either the presence of a viscoelastic environment, ${ }^{70}$ or of a crowded environment with random obstacles that are not interacting with each other. ${ }^{71-73}$ These are properties of the system that seem highly consistent with the environment within the liquid cell. The analysis shows that at the lowest dose rate $\left(1.6 \mathrm{e}^{-/} / \AA^{2} \mathrm{~s}\right)$, a significant number of micelles (10) are either partially or fully trapped for extended periods of time, which is not seen at higher dose rates. For increasing dose rates, a larger fraction of micelles follow a consistent sub-diffusive motion, with exponents $(a)$ that are larger than for lower doses. However, at all dose rates, the micelles' motion is generally sub-diffusive, and to the same degree $\left(\mathrm{D}_{a} \sim 10^{3}-10^{4} \mathrm{~nm}^{2} / \mathrm{s}^{a}\right.$, Table S5), with clear variations (turning points) in their sub-diffusion exponents $(a)$ as a
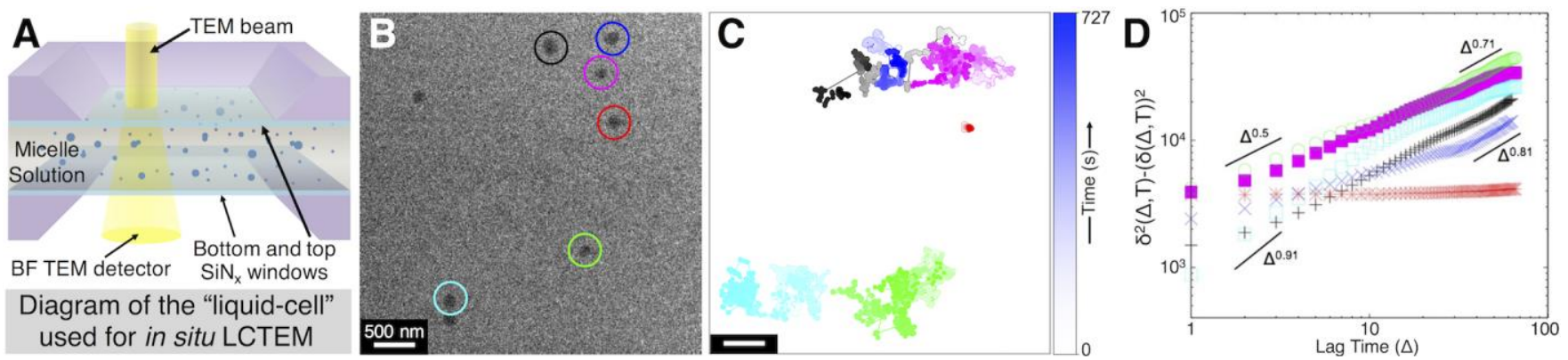

Figure 2. Analysis of micelle motion in solution by LCTEM videography. (A) Schematic of liquid-cell enclosure. (B) Single frame from one LCTEM video of micelle dynamics (Video $\mathrm{S} 2$, dose rate $=1.6 \mathrm{e}^{-} / \AA^{2} \mathrm{~s}$ ). (C) MOTA $\mathrm{x} / \mathrm{y}$ position trajectory over the duration of the $727 \mathrm{~s}$ LCTEM video). Scale bar is $500 \mathrm{~nm}$. (D) Variance of the micelle displacement (time-moving average variance of displacement, TMAVD) as a function of lag time, $\Delta$, for each of the six color-marked micelles. The indicated slopes mark inflection points in the profiles. The red micelle is essentially immobilized ("trapped") a $\mathrm{SiN}_{\mathrm{x}}$ window surface for the duration of LCTEM observation. 
functions of lag time. We also note that cross-correlation analysis (Figure S5) of particle surface area (s) and step size (1), shows there is no relation between each individual micelle's dynamics and its average size or any changes in its size.

Some local "trapping" events do occur at all doses, likely due to surface interactions, and are described by a distribution of waiting times between Levy jumps. See for example the particle circled in "red" in Figure 2B-D, as opposed to the mobile particles in that same field of view. This particle moves very little, and in a very confined fashion as subtle lateral vibrations, while it is trapped throughout the time course observed. Micelles that move within the liquid cell between trapping periods perform $\mathrm{fBm}$ and experience only random obstacles characteristic of the confined volume (other micelles and/or brief window interactions). The $\mathrm{e}^{-}$beam does not interact directly with the micelles, but larger dose rates provide a more probable escape from traps, likely by altering the charge of either the window surface or the micelle, ${ }^{74}$ or by slightly altering the local solution chemistry and disturbing micellesurface adsorption forces. Detachment of the micelle from the window surface occurs via a jump into the "bulk," where it proceeds to move "normally" by sub-diffusive fBm. Overall, these data suggest that although the beam does not directly affect the motion of the micelles, secondary effects, such as beam induced changes in how the micelles interact with the surface or possible beam induced changes in solution chemistry, are present. ${ }^{61,75}$ These secondary beam effects could also play a role in the evolution processes that we have also observed, but we note that no differences in motion-character were found between micelles that actively evolve, undergoing fusion and growth, and those that simply migrate within the confines of the liquid cell. Additionally, similar evolution processes are observed at all the dose rates used. However, the ADOMA data, which find clear sub-diffusive, constrained motion of the LCTEM micelles, do make it clear that these micelles are continually interacting with the enclosing membranes. No location in the liquid cell is ever more than $\sim 500$ $\mathrm{nm}$ away from one of the solid interfaces. This high-degree of surface interactions create a local environment experienced by each micelle in the liquid-cell that differs from that which is experienced by the average micelle in the bulk solution that is enclosed within a large glass vial at quiescent conditions, where on average, micelles experience negligible interactions with the glass surface of the enclosure due to the very low surface area per volume. Epps el $a l .^{30,31}$ and others have found evidence that the evolution of kinetically trapped micelles is often facilitated by interactions at an interface. The solidliquid interface interactions imposed by the liquid-cell enclosure could have played a role in "triggering" the evolution of the kinetically-trapped micelles in this study, which have al-

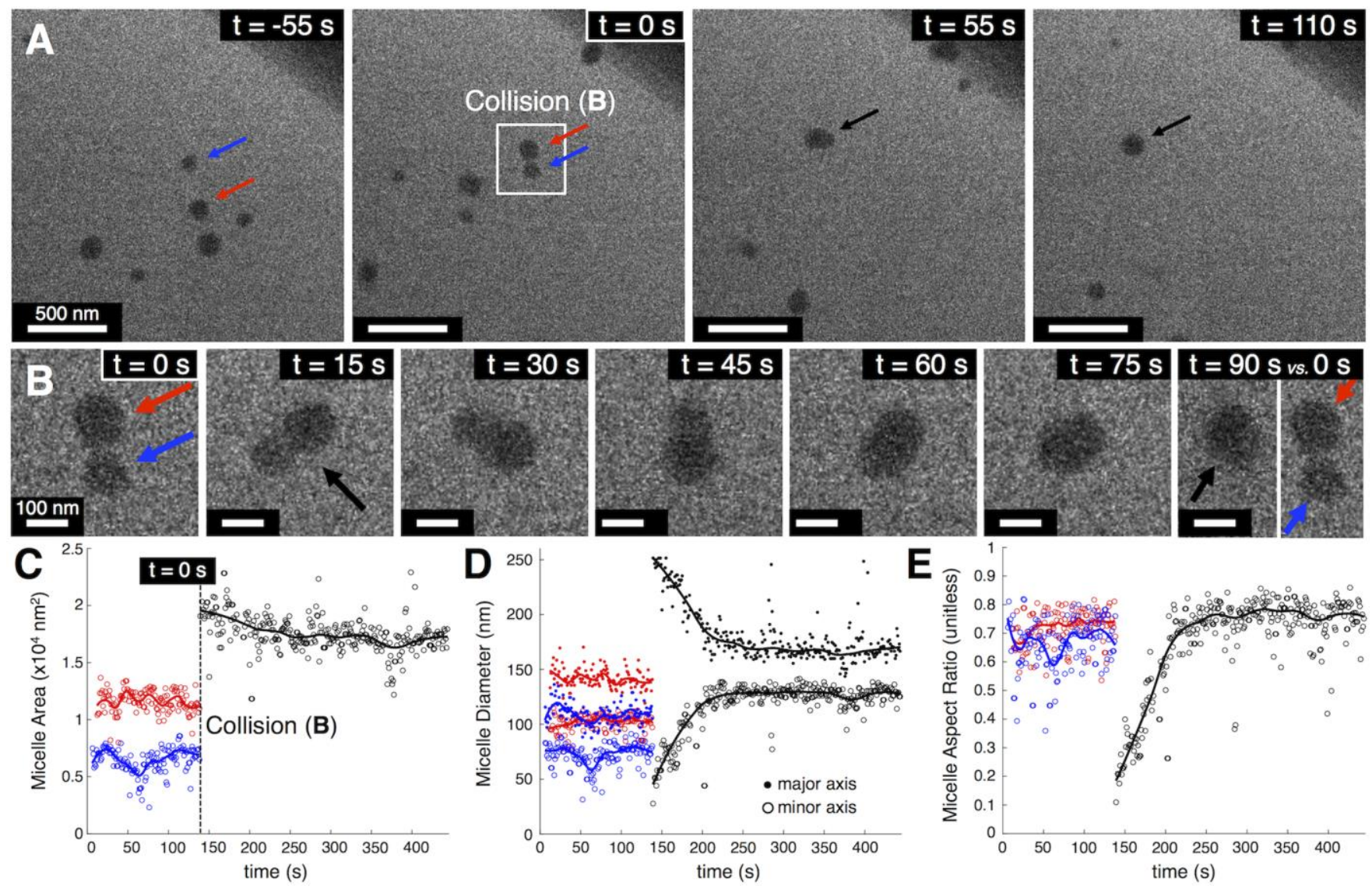

Figure 3. Micelle-micelle fusion event captured by LCTEM. (A) Single frames (time-lapse) from one in situ LCTEM video (6 e $/ \AA^{2} \mathrm{~s}$, Video S3) beginning at $\mathrm{t}=-55 \mathrm{~s}$ (prior to collision). The video frame where collision between the red and blue micelles first occurs is referred to as " $\mathrm{t}=0 \mathrm{~s}$ " (start of fusion-relaxation process). Frames shown labeled as $\mathrm{t}=55 \mathrm{~s}$ and $110 \mathrm{~s}$ show relaxation yielding a single assembly (black-labeled micelle). (B) Zoom-in beginning at $\mathrm{t}=0 \mathrm{~s}$, showing magnified time-lapse images of the of the fusion-relaxation process between the red and blue micelles. (C) Micelle projection-area plot, (D) micelle major (closed circles) and minor (open circles) projectiondiameter plot, and $(E)$ micelle aspect ratio ( $\left.\mathrm{D}_{\text {minor: }} \mathrm{D}_{\text {major }}\right)$ plot for the red, blue and black micelles over the duration of the LCTEM video observation. 
ready undergone evolution and growth within the liquid-cell prior to any $\mathrm{e}^{-}$beam irradiation (Video $\mathrm{S} 1$ and Fig. S4). Awareness of the nature of the liquid-cell environment and potential secondary effects from $\mathrm{e}^{-}$beam irradiation in relation to the observed micelle growth, evolution, collisions, and fusion is key for meaningful interpretation of the LCTEM video data.

Size Evolution by Micelle-Micelle Fusion Observed by LCTEM. In the LCTEM video data, multiple explicit micellemicelle fusion-and-relaxation events occur, which have been directly captured on the nanoscale. One such example of unambiguous micelle-micelle fusion is shown in Figure 3 (Video S3). A series of LCTEM video frames are shown in (Figure 3A,B) with plots displayed for the MOTA-measured projection areas (Figure 3C), minor- and major-axis diameters (Figure $3 \mathrm{D})$, and elliptical aspect-ratios $\left(\mathrm{D}_{\text {minor }}: \mathrm{D}_{\text {major }}\right)$ (Figure $3 \mathrm{E}$ ) for the two fusing micelles (red and blue) and post-fusion assembly (black). Prior to collision, the red- and blue-labeled micelles are separated by several microns in lateral distance and are moving normally for the system (discussed above, $\mathrm{fBm}$ driven by fGn). Both objects have similar oblongspherical morphologies in projection (aspect ratios $\sim 0.7$, consistent with the average for all micelles observed, Figure S6). Simulations also suggest $\sim 0.7-0.8$ average aspect ratios are the inherent projected-shapes of these micelles in solution (discussed below). After several minutes of continuous monitoring, the two micelles randomly approach, and collide, initiating a clear fusion event (Figure 3A,B). The process begins through contact of the hydrophilic coronas of the two micelles, initiating neck formation. Despite this unprecedented view of a nanoscale particle fusion process, the molecular details related to chain re-organization cannot be resolved in LCTEM video frames, leading to our analysis of these processes through in silico modeling (vide infra). Immediately following this collision (marked $\mathrm{t}=0$ in Figure 3A,B), the coalesced aggregate ("black") undergoes a process of morphological relaxation over the subsequent $\sim 120 \mathrm{~s}$, as its projected area
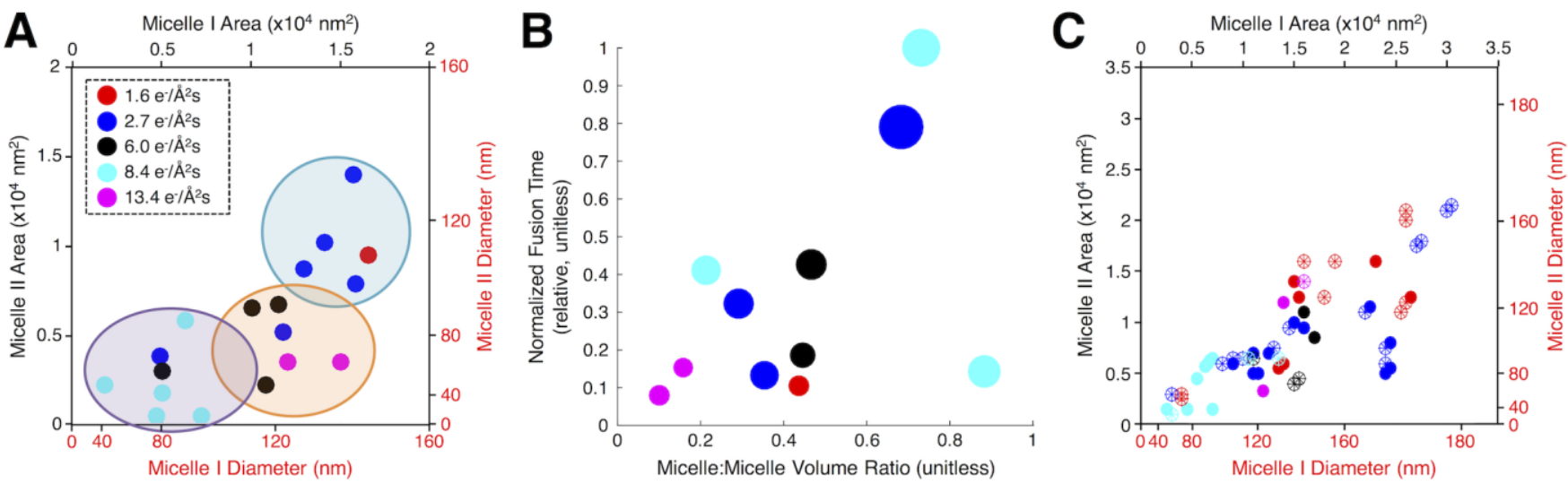

Figure 4. Statistical analysis of micelle-micelle fusion events and non-fusion collisions observed by LCTEM. (A) Scatter plot of all the observed LCTEM micelle-micelle fusion events (18) as a function of the pre-fusion micelle projection area (and average diameter, as the mean of the major and minor diameters) of the two micelles involved, where "Micelle I" is selected as the larger of the two fusing micelles (Table S3). Purple, orange, and teal rings indicate three general micelle-micelle size regimes (small-small, small-large, and large-large, respectively) for observed micelle fusion events. Fusion events are given as solid, colored circles indicating the dose rates at which they occur per the key. (B) Scatter plot of the fusion-relaxation time (normalization details in Supporting Information) for each micelle-micelle fusion event followed to complete relaxation (11) as a function of the volume ratio of the pre-fusion micelle-micelle pair. The size (area) of each circular data-point is displayed as proportional to the percent volume increase for that fusion event (i.e. larger \%vol. increase represented by proportionally larger circle). Colored circles, match dose rate key in (A). (C) Scatter plot of the 58 micelle-micelle collisions or periods of overlap that do not result in a fusion event, as a function of the projection area (and average diameter) of the two colliding micelles. Closed circles indicate collision/overlap events that last less than $6 \mathrm{~s}$ (brief interactions), and open circles indicate collision/overlap events that last longer than $10 \mathrm{~s}$ (prolonged interactions). The color of each data-point in (A,B,C) again indicates the dose rate (key in (A)). 
ed and discussed below, such multi-micelle processes are indicative of the kind of complex interactions inherent to this system and to evolution of soft matter in solution more generally.

Overall, the relaxation times (time for aspect ratio to reach and stabilize at $~ 0.7$ ) of fusing micelles show a loose dependence on volume. That is, fusion events between assemblies with larger volumes, as projected by their external surfaces, have on average longer relaxation times (Figure 4B, Table S3), in general agreement with the classical Frenkel viscous flow model for droplet fusion and the Koch-Friedlander (KF) solid-state diffusion model for particle coalescence. ${ }^{76-79}$ However, in these data, the size-ratios have much less influence over the fusion relaxation times than is predicted by the classical fusion models. Relaxation in the viscous flow and solidstate diffusion models is primarily driven by neck-curvature stress gradients and the large surface-to-bulk energy ratio due to asymmetry, impelling the reduction in specific surface area, and resulting in a strong relationship between the relaxation time and the size-ratio of the two fusing objects. That is, for fusion events involving the same total number for atoms, significantly faster relaxation is expected for extremely unequalsized particle pairs, and the slowest relaxation time for perfectly equal-sized pairs. ${ }^{77,79}$ The LCTEM micelle fusion data, which lack this classical dependence on size ratio, suggest that the kinetic barriers and pathways of amphiphilic BCP micelle fusion-and-relaxation must be influenced by additional physical/chemical parameters that alter the driving forces leading toward local energy minima, such as the complex, amphiphilic phase-separated internal structure (unimer and water) of each of the fusing assemblies, the process of internalization of external water molecules during fusion, or the transport of internal water pockets. From the LCTEM data, we hypothesize that each micelle's internal structure and its orientation at collision, related to possible surface "hot spots" or localized corona inhomogeneities, to be important factors that govern micelle fusion and relaxation, and in collisions that do not result in fusion. This morphological and orientational influence on the outcome of collision-and-fusion seems analogous to the importance of the unique crystal structure and lattice-orientation of each particle in metal nanoparticle fusion, where orientedattachment is well documented, and is known to heavily influence both the coalescence time and the final particle morphology, such as defects, twinning, and surface facets. ${ }^{80}$

Volume increase (generally $\sim 15-50 \%$ ) associated with fusion is found in all the LCTEM observed micelle fusion events, with the extent of increase varying for each specific event (Table S3 and Figure 3B). This finding indicates that though the total number of BCP chains can be assumed to remain essentially constant before and after collision, the distribution of polymer chains and the volume of internal water, in the post-fusion assemblies have reorganized into morphologies that do not directly mirror either of the pre-fusion micelles' morphologies, and cannot be modelled in their fusionbehavior as solid, homogenous droplets or particles according to classical fusion theories. Interestingly, the most equal-sized micelle pairs generally experience the largest volume increase. However, fusion events with longer relaxation times also generally result in a larger volume increase, making it impossible to determine whether it is the longer fusion-relaxation time for more equal sized particles that enable a greater amount of solvent incorporation, or that for certain fusion events, which happen to involve more equal-sized micelles in our observations, a greater amount of solvent is incorporated, slowing the relaxation and reorganization process.

Non-Fusion Collisions Observed by LCTEM. Frequently collisions, or "proximity interactions", did not result in fusion, where instead micelles might collide and quickly separate, or remain close or touching for extended periods with what appear as collaborative motion before eventually separating (Figure 4C). Non-fusion inducing interactions are frequent (fusion ratio of $\sim 1: 3$ ), and occur for the entire size range of micelles. For theses micelles, collision and fusion is not prevented by potential barriers to close-approach or collision as has been previously suggested, attributed to strong Coulombic repulsion between ionic micelles or steric repulsion between nonionic micelles. ${ }^{17,18,35,81-83}$ For the BCP studied, the charge neutral nature of the corona block potentially reduces ionic repulsion compared to charged corona species ${ }^{84-87}$ and the short length of the corona block relative to the core block could reduce potential steric hindrances to fusion. ${ }^{88-90}$ The ADOMA trajectory analysis for micelles involved in collisions does not find any detectable differences in the pre-collision motion behavior for events that result in fusion and those that do not. However, such analysis is limited in its precision due to the frame rate ( $1 \mathrm{fps}$ ) of the LCTEM videos relative to the temporally brief pre-collision period of interest, which is only several seconds. Furthermore, we note that there are no statistically significant deviations in the measured micelle area directly associated with closely interacting, or colliding particles where fusion does not occur. Certainly, exchange of polymer between micelles is a well-documented phenomenon in many
A

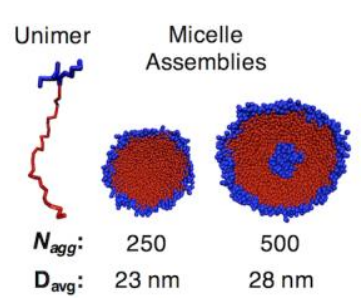

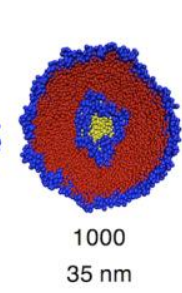

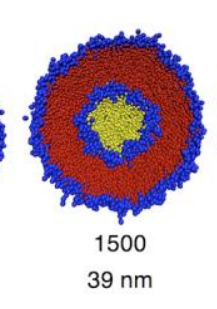

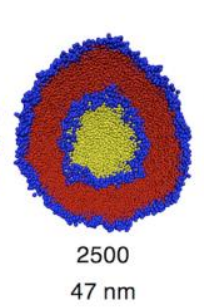
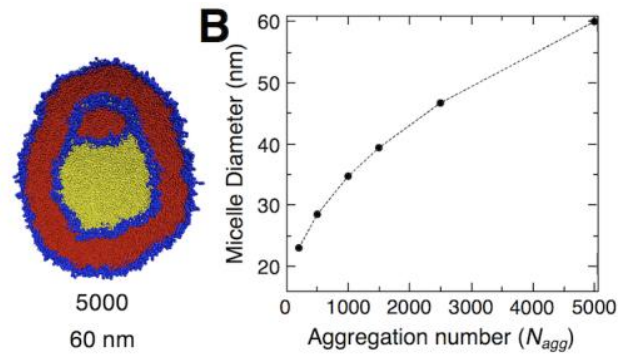

Figure 5. As assembled structures spontaneously formed from unimers in silico. (A) Simulation-generated morphologies of assemblies for increasing $N_{\text {agg. }}$. Left-most is the coarse-grained model of a single polymer chain (unimer) of the structure shown in Figure 1A. Each subsequent image shows the cross-section through the middle of the macromolecular aggregate once it has stabilized after assembly. Blue represents the hydrophilic peptide block, reds represents hydrophobic phenyl, and yellow represents the solvent molecules (water). For clarity, only the solvent trapped inside the structures is shown (the surrounding solvent molecules have been removed). (B) The average external surface diameter $\left(\mathrm{D}_{\mathrm{avg}}=\left[2 \times\left(\left\langle\mathrm{R}_{\mathrm{g}}{ }^{2}\right\rangle\right)^{1 / 2}\right]\right)$ for each assembled and stabilized micelle (values also listed below each simulation image in $\left.(\mathrm{A})\right)$. 
equilibrium systems. ${ }^{3,9,41,52,91,92}$ However, our LCTEM observations of these collisions cannot confirm or rule out exchange as we see no evidence of nanoscale changes in the particles, and we cannot resolve individual polymer chains in solution.

In silico Simulations of Micelle Assembly, Collisions, and Fusion. To gain insight into the molecular transport and organization processes that can only be inferred from the lowcontrast 2D projection LCTEM videos, We sought to couple experimental observations with dynamical simulations of a coarse-grained model of the amphiphilic block copolymer system studied experimentally by LCTEM. ${ }^{93-96}$ The coarse grained system contains information regarding the molecular architecture of the macromolecule, with chemical details encoded into generic intermolecular interaction parameters (Supporting Information), and has been applied previously for modeling bilayer membranes. ${ }^{93-100}$ First, we simulated the assembly of the macromolecules for increasing aggregation number, $N_{a g g}$ (Figure 5 and Figure S8), generating a library of stable micelles, from small crew-cut micelles to larger vesicles (polymersomes) formed for different $N_{\text {agg, }}$, which we used to simulate the processes of collision and fusion (Videos S12S15, Figure 6 and Figure S9). ${ }^{39}$ The size of the micelles used in these simulations was limited to the smallest micelles observed in the LCTEM experiments (diameters up $\sim 60 \mathrm{~nm}$ ), but the lowest $N_{\text {agg }}$ (250) assembly was very similar to initial kinetically-trapped micelles ( $20 \mathrm{~nm}$ diameter) as imaged by cryo-TEM (Figure 1A).

We start by simulating collisions between the small assemblies $\left(N_{\text {agg }}=250\right)$, where the fusion process is rapid in silico and yields simple spherical micelles with a homogeneous distribution of unimer throughout (Figure 6A, Video S12 and Figure S9A), and continue on to larger sizes, investigating both matched and mismatched fusion-pairs (Figure 6B-D). For low- $N_{\text {agg }}$ assemblies (250), no volume change due to external water internalization occurs. We note that in the LCTEM data

A

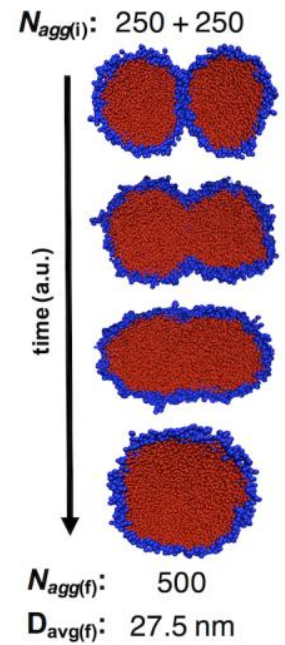

B

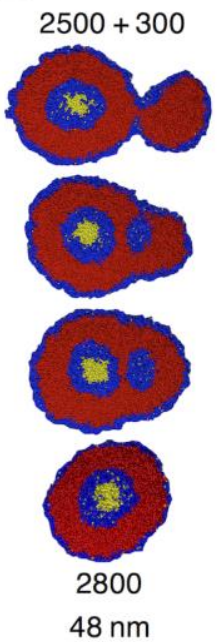

C

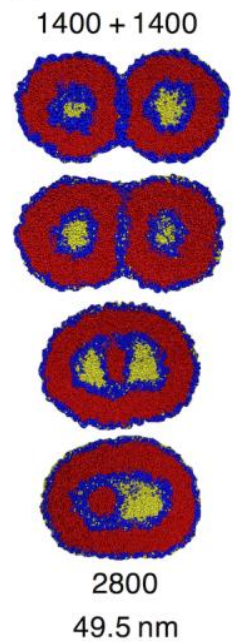

D

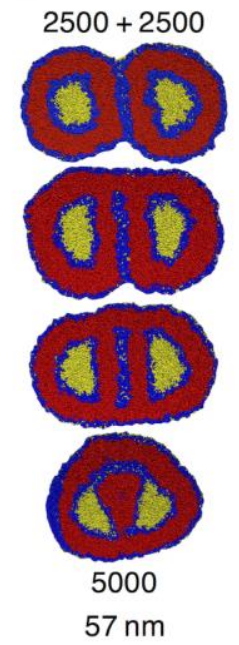

we have not captured any fusion events involving micelles of this small size that could be quantified, though the size of these spherical micelles is very consistent with the initial prepared micelles (Figure 1A). The speed of these fusion simulations relative to those of larger assemblies does suggest that the small kinetically trapped morphologies could rapidly transition into larger sizes, with these larger sizes more easily captured during our LCTEM observations.

Fusion between a large vesicle $\left(2500 N_{a g g}\right)$ and a small spherical micelle (300 $N_{\text {agg }}$ ) is more complex (Figure 6B, Video S13 and Figure S9B), beginning with the formation of a "hemifusion stalk" on the contact interface, and the entrapment of an internal double-bilayer polymer structure and an additional solvent pocket. This intermediate morphology evolves by combining the temporarily internal double-bilayer structure with the surface bilayer of the vesicle as the "new" solvent pocket collides and amalgamates with the larger preexistent solvent pocket. The final micelle $\left(N_{a g g}=2800\right)$ adopts a classical vesicle structure, very similar in both morphology and size to that of an equivalent micelle formed by selfassembly alone (Figure 5A). As the assembly relaxes from being highly elliptical following contact and the beginning of fusion, the aspect ratio (Figure $6 \mathrm{E}, \mathrm{D}_{\text {minor }}: \mathrm{D}_{\text {major }}$ ) steadily increases then plateaus $(\sim 0.75)$, decreases slightly, before finally increasing stabilizing at a value of $\sim 0.8$, similar to the slightly elliptical morphologies observed by LCTEM of freely diffusing individual micelles (see above). These in silico data provide some insight into molecular-scale dynamics occurring during the necking- and-relaxation processes observed in LCTEM. Indeed, the model predicts solvent encapsulation during fusion and phase transition; a process consistent with direct experimental observation of volume increases accompanying micelle fusion (see above).

For direct comparison, and to test our conclusions from the LCTEM fusion data, we next simulated a pair of micelles with

Figure 6. In silico fusion simulations of particles of different aggregation number $\left(N_{\text {agg }}\right)$ and diameter (Davg). (A-D) Time-series of frames from fusion simulations for different colliding micelle-micelle pairs. (top row) Frames at time of initial collision. (bottom row) Frames of the post-fusion micelle after relaxation. The four columns of images are not displayed at the same magnification or with the same time intervals between frames. (A) (Video S12) (250-250) The fusion of two small spherical "crew-cut" micelles, (B,C), fusion of larger aggregates where, one, (B) (Video S13) (2500-300) or both (C) (Video S14) (1400-1400) are polymersomes. (D) (Video S15) (2500-2500) shows the fusion of two larger polymersomes (on the same diameter length-scale observed by LCTEM). The final $N_{a g g}$ and external-surface diameter of the bottom row micelles (fully-relaxed) are given below each time-series. (E) Aspect ratio $\left(\mathrm{D}_{\text {minor }}: \mathrm{D}_{\text {major }}\right)$ plots of the fusing micelles (post-collision) from the four fusion event simulations in (A-D). The line color that corresponds with each fusion event is displayed in the legend (micelle-micelle $N_{\text {agg }}$ ratio). The inset shows the same plot in log-scale of simulation time (x-axis) for the first 3000 simulations frames. Transmission visualization for simulations in (A-D) are shown in Figure S9. 
the same total $N_{a g g}(2800)$, but where the size-ratio is equal rather than highly asymmetric as in the previous simulation. Fusion between two equal-sized vesicles both $1400 N_{\text {agg }}$ (Figure 6C, Video S14 and Figure 9C), follows a different evolution pathway, and gives rise to a more complex, higher-order micellar morphology post-relaxation, containing both internal water pockets and an internal polymer "worm" within the vesicular surface-bilayer. In this simulation, the post-fusion micelle equilibrates to a lower aspect ratio than the previous case $(\sim 0.73$ vs. $\sim 0.8$ ), and does not exhibit a 2-step behavior. Furthermore, the amount of water internalized during fusion is detectably larger than for the previous $N_{\mathrm{agg}}=2800$ fusion structure (Figure 6B), but full relaxation occurs more rapidly (Figure 6E inset). We also note that the relaxed assembly here (Figure 6C), also contains more internal water than the 2800 $N_{\text {agg }}$ vesicle formed by self-assembly alone (Figure 5). These findings are consistent with the LCTEM data, where fusionrelaxation time does not have the classical relationship (KF fusion model) to the size-ratio, where the symmetric pair would be expected to fully relax more quickly. These data also suggest that the relationship between volume increase and relaxation time during fusion are strongly influenced by the structure of each micelle involved (vesicle vs. solid-sphere). In this manner, we hypothesize that micelle structures that are the product of evolution by fusion have memory; their current structures are not only reflections of their $N_{a g g}$ and the local solution environment but also the specific path they took during formation.

In the final simulation, we investigated the fusion process

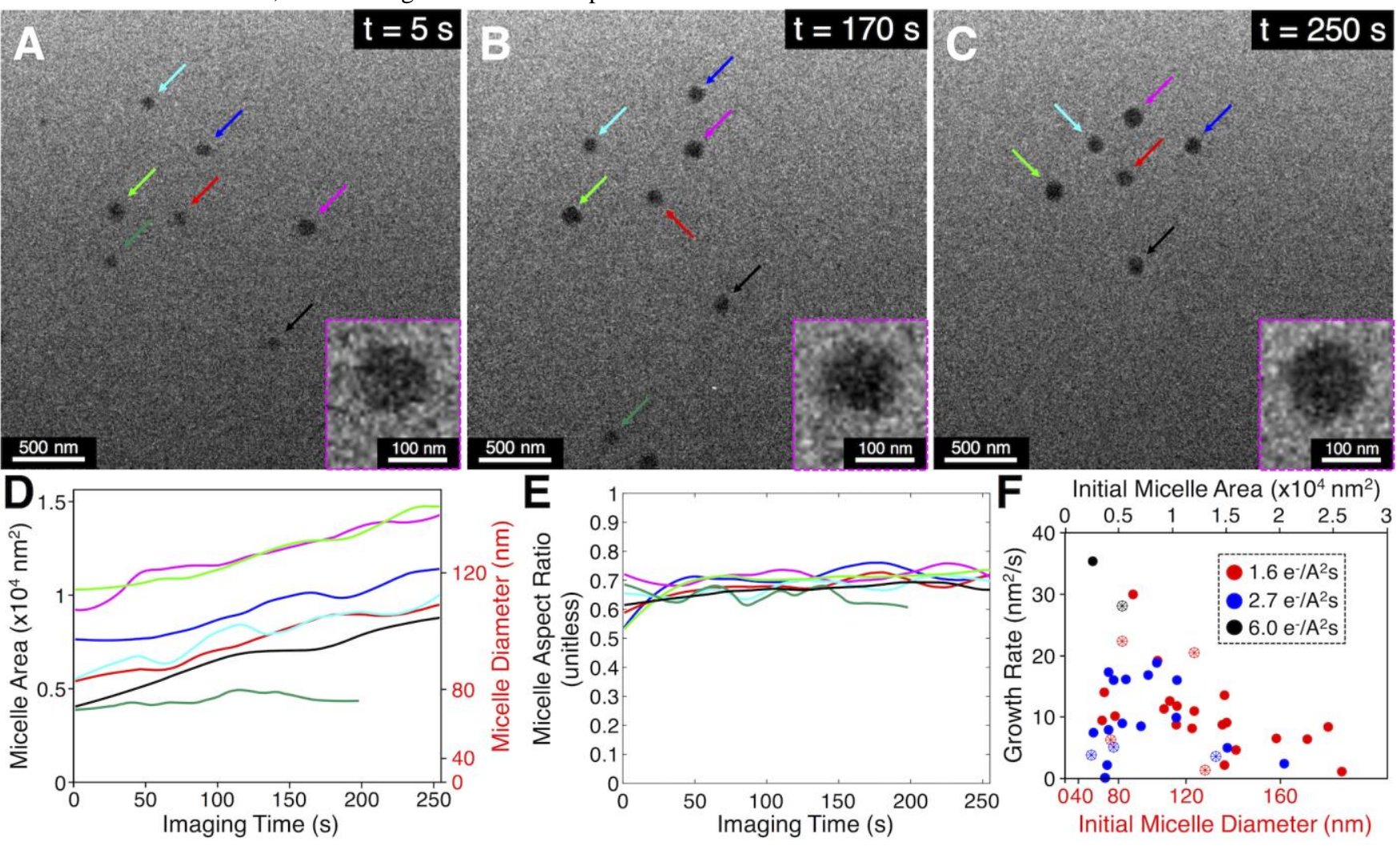

for collisions events involving even larger, equal-sized, vesicles $\left(N_{\text {agg }}^{1}=N_{\text {agg }}^{2}=2500\right.$ ) (Figure 6D, Video S15 and Figure S9D), where relaxation proceeded through even more intricate pathways than for the smaller vesicles and spherical micelles (a detailed discussion of these simulation data is in the Supporting Information). Most notably, relaxation occurred by a 2 -step process not observed for the other fusion events investigated for equal-sized pair (Figure 6E), first temporarily stabilizing at $\sim 0.67$ aspect ratio, before rapidly transitioning (at $\sim 1400$ simulation frames) and relaxing at its final aspect ratio of $\sim 0.8$. This second relaxation step is considerably more severe than that for the asymmetric pair in Figure 6B. After full relaxation of the external surface, when the projection aspect ratio remains essentially constant, the internal morphology is tortuous and fluctuating, with multiple internal solvent pockets remaining spatially separated but locally mobile (Video S15). Comparing the morphology resulting from fusion with that formed by self-assembly alone $\left(N_{a g g}=5000\right.$, Figure 5), we find that both have complex internal morphologies containing polymer and solvent phases. However, several differences differentiate these two assemblies, such as the discrepancy in the number of separate internal-water pockets, in the tortuosity and morphology of the internal BCP phases, and in the external-surface diameters and volume of internal water. These simulations demonstrate the complex interplay between the drive towards thermodynamic equilibrium and the kinetic constraints during formation depending on the pathway taken.

Individual Micelle Growth Observed by LCTEM. In ad-

Figure 7. Micelle growth by unimer attachment. (A-C) Time-lapse of individual frames from one LCTEM video $\left(2.7 \mathrm{e}^{-} / \AA^{2} \mathrm{~s}\right.$, Video S6) where individual micelles grow without fusion. Colored arrows track the location of each micelle over time. The purple-marked micelle is displayed at higher magnification in the insets. (D) Micelle area (and average diameter) plot, (E) micelle aspect ratio plot ( $\mathrm{D}_{\text {minor: }} \mathrm{D}_{\text {major }}$ ), and (F) scatter plot of the average linear-fit growth rate of 51 micelles observed, as a function of the initial micelle size (area and average diameter) (Table S4). Data points with empty circles indicate micelles that exhibit some periods of size reduction, but where overall the increase in size. The color of each data point indicates the dose rate experienced by that micelle. 
dition to size evolution by micelle-micelle fusion, micelle growth in the absence of fusion is abundant in the LCTEM data (Figure 7). We attribute this growth to a process of unimer attachment and insertion of solvated, free polymer chains, or small clusters of polymer, into the micellar assemblies. ${ }^{3,9,41,43-45,52,82}$ The origin of these free unimer (or small clusters), which are not resolvable in the LCTEM videos, could be the surface of the liquid-cell windows, where the concentration of adsorbed polymer-chains could be significantly greater than the bulk critical micelle concentration (CMC), driven by the strong unimer-surface affinity from van der Waals forces, hydrogen bonding and Coulombic interactions with the plasma-treated $\mathrm{SiN}_{\mathrm{x}}$ surface, and the large hydrophobic block of these amphiphilic unimers that would favor aggregation at surfaces. ${ }^{101,102}$ Though these micelles are stable at quiescent conditions, ${ }^{103}$ it is likely that the very large surface area of the $\mathrm{SiN}_{\mathrm{x}}$ windows (relative to the "bulk" solution) in the liquid-cell has generated high concentrations of "free" unimer accumulated at the window surfaces. ${ }^{104,105}$ Indeed, interfaces promoting phase transitions and growth have been observed for polymeric amphiphile assemblies. ${ }^{30,31}$ Physical perturbation during the liquid-cell assembly process, either induced by local compressive pressure or shear forces applied during loading, or slight variation in solution concentration during loading the very small volume of sample solution, could also play a role. 7,30,31,106 As discussed above, the ADOMA results suggest that secondary $\mathrm{e}^{-}$beam effects are operative in these LCTEM experiments. Charging of the $\mathrm{SiN}_{\mathrm{x}}$ membrane and/or the adsorbed unimer chains by the $\mathrm{e}^{-}$beam during LCTEM imaging was likely to have induced these unimer to release from the surface into solution at local concentrations greater than the CMC of the bulk solution, driving the free unimer to aggregate into the local micelles, which we observe growth as individual micelle growth absent of collisions.

Micelles monitored by LCTEM exhibit continuous, gradual individual growth over time (linear area-growth rate of $15 \pm 2$ $\mathrm{nm}^{2} / \mathrm{s}$ in Figure 7A-D, Video S6; growth rates of all micelles in LCTEM data in Table S4), and maintain essentially constant projection-morphologies during this time (aspect ratios of $\sim 0.7$ continuously, Figure 7E). Interestingly, as this LCTEM video (Video S6) proceeds beyond the first $250 \mathrm{~s}$ that are displayed in Figure 7A-E, two distinct fusion events occur involving micelles that had been previously growing in isolation in the absence of collisions or fusion. The two evolution pathways, growth via unimer attachment and growth via micelle-micelle fusion, are both at work for individual micelles and for local ensembles of micelles.

Using MOTA, we have tracked and quantified the projected area over time for 51 micelles that were continually imaged for longer than $180 \mathrm{~s}$, sampling all the LCTEM videos (Videos S1-S11). Average growth rate for each micelle is plotted as a function of micelle size (projection area and average diameter) in Figure 7E (Table S4). There is an overall inverse trend correlating growth rate and micelle size. The average unimer attachment growth-rate slows as micelles become larger, possibly reaching an equilibrium size regime where additional growth is unfavorable and is essentially arrested. We lack sufficient LCTEM data to quantify an asymptotic micelle size maximum with certainty. No micelle larger than $\sim 180 \mathrm{~nm}$ diameter was observed at the start of the $\mathrm{e}^{-}$beam illumination for any LCTEM video, and the largest final micelle at the end of any LCTEM video was $\sim 190 \mathrm{~nm}$ diameter. Growth by free- unimer attachment appears to remain a viable pathway for the largest micelles (up to $\sim 180 \mathrm{~nm}$ ), while fusion is less favorable. It is possible that these large micelles can undergo fusion events either with small micelles or other large micelles, events that have simply not been captured during our LCTEM experiments. However, the lack of observed fusion events involving the largest micelles in comparison to the numerous fusion events observed amongst micelles below $\sim 160 \mathrm{~nm}$ diameter, suggests a low to nil probability of such events occurring, further supporting our conclusion that micelles above this size ( 200 nm diameter) are approaching a stable, equilibrium assembly size for this polymer within the environmental conditions of the liquid-cell.

\section{CONCLUSION}

In the studies presented, liquid-cell (LC)TEM has provided an unprecedented window through which to directly observe the diffusion behavior and morphological transitions of amphiphilic polymeric micelles in water. Detailed analysis of the diffusive motion reveals complex, non-Brownian behavior and mechanisms, with indirect effects from the $\mathrm{e}^{-}$beam at the low dose-rates used. Micelles were found to interact and collide with a variety of results, including quickly deflecting, extended interactions and separations, and most significantly, evidence for unimer exchange driven growth, and particleparticle fusion and relaxation. Analysis of fusion events by the examination of LCTEM video data combined with in silico modeling of the pathways involved, revealed processes consistent with external-solvent entrapment during fusionrelaxation and the generation of higher-order amphiphile assemblies including complex vesicles and biocontinuous micelles formed from what were initially kinetically-trapped spherical micelles. These findings bring to light the involvement of additional kinetic constraints on fusion-relaxation for collision events involving large, complex morphologies. The direct observation of micelle-micelle fusion, and unimer attachment processes by LCTEM should establish this method as the most direct way of observing processes resulting in phase transitions and for testing viable evolution pathways for soft matter assemblies, complementing commonly used static and/or indirect techniques.

When properly applied, low-dose LCTEM will prove invaluable for confirming transitions observed in bulk solution scattering studies and snapshot analysis by conventional TEM observations. The introduction of the time domain and videographic observation of soft matter nanomaterials and their complexities will be a critical part of their development and functional optimization, and in deepening our understanding of fundamental nanoscale processes in such systems. Furthermore, we propose the observation and analysis techniques presented will be generally useful for examining a broad range of systems including other classes of nanoparticles and nanoscale materials.

\section{METHODS}

Block Copolymer Micelle Preparation: Norbornenyl- $b$ phenyl monomer $(0.054 \mathrm{mmol}, 20$ equiv) was added to a solution of Grubbs' modified second generation catalyst ${ }^{51}$ [(H2IMES)(pyr)2(Cl)2Ru=CHPh] $(0.0027 \mathrm{mmol}, 1$ equiv $)$ in degassed dry DMF and stirred for 20 minutes. The norbornenyl- $b$-peptide monomer ( $0.00814 \mathrm{mmol}, 3$ equiv.), which 
was dissolved in minimal amounts of DMF, was added to the reaction. Then 1 equivalent of norbornenyl-ethanolamine monomer was added every 36 seconds to incorporate the monomer as a blend copolymer. After the last addition, the polymer was left to stir for 20 minutes then quenched with 20 $\mathrm{uL}$ of ethyl vinyl ether and stirred for 1 hour. The polymer was precipitated in ether. The polymer was collected by centrifugation and dissolved in a water-acetonitrile mixture and lyophilized overnight. The polymer was characterized by SECMALS. Phenyl block: $M_{w}-11,900 \mathrm{~g} / \mathrm{mol}, Ð-1.003$; Total polymer: $M_{w}-17,640, Ð-1.040$. The degree of polymerization of the phenyl block (hydrophobic, $m$ in Figure $\mathrm{S} 1$ ) is $D P_{m}$ $=47$, and the degree of polymerization of the peptide-cohydroxyl block (hydrophilic, $n, o$ in Figure $\mathrm{S} 1$ ) is $D P_{n+o}=4$. For a scheme showing the polymer (phenyl- $b$-peptide-cohydroxyl ) synthesis procedure see Figure S1.

The polymer was dissolved in $2 \mathrm{~mL}$ of DMF at $2 \mathrm{mg} / \mathrm{mL}$, then $2 \mathrm{~mL}$ of water was added to the rapidly stirring polymer solution at $0.1 \mathrm{~mL} /$ hour. After complete water addition, the solution was transferred to 10,000 molecular weight cut off dialysis tubing and dialyzed into Dulbecco's phosphatebuffered saline (DPBS) for 3 days with 3 DPBS changes. The micelles were then filtered using 1 micron filters and analyzed by dynamic light scattering (DLS) data using a Wyatt NynaPro Nanostar (Wyatt Technology Corp., Santa Barbara, CA) instrument. This initial micelle solution was later used for the in situ LCTEM experiments, the bulk perturbation experiments, and the cryo-TEM imaging-characterization experiments that we report in this study.

Liquid-Cell Assembly: LCTEM experiments were performed using a Hummingbird Scientific (Lacey, WA) LiquidFlow TEM Holder with the Dual-Flow Mixing Tip. The two inlet lines and single outlet line were prefilled with DPBS buffer. Two Norcada Inc. (Edmonton, AB, Canada) $30 \mathrm{~nm}$ thick $\mathrm{SiN}_{\mathrm{x}}$ membrane chips $(50 \mu \mathrm{m}$ x $50 \mu \mathrm{m}$ lateral window dimensions) were glow discharged for $60 \mathrm{~s}$ at $10 \mathrm{~mA}$ using an S4 Emitech K350 instrument (Quorum Technologies Ltd., Kent, United Kingdom). With one chip $\left(\operatorname{SiN}_{x}\right.$ membrane side facing up) in the holder tip, one $0.85 \mu \mathrm{L}$ droplet of BCP micelle solution was applied to the chip surface using a micropipette. The second chip ( $\mathrm{SiN}_{\mathrm{x}}$ membrane facing down) was placed over the sample droplet and the bottom chip, and the top and bottom chips were properly aligned manually with tweezers. An additional 1.2 $\mu \mathrm{L}$ droplet of BCP micelle solution was then pipetted into the side channel of the holder tip. The over-clamp of the tip was put into place, and the slide plate was pushed into position to seal the tip assembly. The liquid-cell integrity was checked by pumping the holder down to vacuum ( $\left.8 \times 10^{-6} \mathrm{mbar}\right)$ in the pre-pump chamber. The holder was inserted into the TEM to begin the LCTEM experiment within $10 \mathrm{~min}$ of loading the sample solution into the liquid-cell holder.

LCTEM and cryo-TEM Microscope Conditions: An FEI (FEI Company, Hillsboro, OR) Tecnai G2 Sphera transmission electron microscope (TEM), operating at $200 \mathrm{keV}$ was

\section{ASSOCIATED CONTENT}

\section{Supporting Information}

The PDF SI file contains the Supporting Methods (cryo-TEM sample preparation, MOTA analysis, quantitative LCTEM data used for the in situ LCTEM experiments and for cryo-TEM imaging, at an emission current of $\sim 4 \mu \mathrm{A}\left(\mathrm{LaB}_{6}\right.$ tip), and using a $2 \mathrm{~K}$ x $2 \mathrm{~K}$ Gatan UltraScan CCD camera and Gatan Digital Micrograph image acquisition software (Roper Technologies, Sarasota, FL). The LCTEM e- dose rate conditions for all Videos and Figures are listed in Table S1 (1.6-13.4 $\left.\mathrm{e}^{-} / \AA^{2} \mathrm{~s}\right)$, and were calculated using the beam current measured by the phosphorous screen of the Tecnai through vacuum (i.e. with the liquid-cell sample rod retracted and OL aperture removed) and the measured beam diameter used for each magnification associated with that LCTEM video/image. The TEM camera was operating in continuous imaging mode ("search") with a single frame exposure time of $0.3 \mathrm{~s}$ at image dimensions of 1024 x 1024 pixels (binning: 2). The Gatan CCD clearing time is $\sim 0.7 \mathrm{~s}$ per exposure, so the actual image-refresh rate (video frame rate) was $1.0 \mathrm{frame} / \mathrm{s}$, but each frame was only acquired over $0.3 \mathrm{~s}$ of exposure. Dose rates in Gy/s (Table S1) were calculated according to Ref. ${ }^{57}$ (Schneider et al.), using a Total Stopping Power value for liquid water at $200 \mathrm{keV}$ electrons (NIST ESTAR database).

Low-dose imaging protocols were used for cryo-TEM imaging to minimize sample exposure to the e- beam prior to the recording of an image at a given area of the sample. Recorded cryo-TEM images were acquired at a maximum dose of $\sim 10 \mathrm{e}$ $/ \AA^{2}$ per image. TEM camera exposure times of 1 or $2 \mathrm{~s}$ were used for all cryo-TEM images, with image dimensions of 2048 x 2048 pixels (binning: 1).

LCTEM Video Acquisition: The raw LCTEM videos were acquired using VLC media player screen capture software, at a frame capture rate of $10 \mathrm{fps}$ (LCTEM CCD camera frame rate is $1 \mathrm{fps}$, which limits the frame rate of the LCTEM videos). The VLC media player (VideoLAN Organization, opensource) screen capture software was directed at the Gatan Digital Micrograph software pane that was continuously acquiring the TEM images from the camera at native resolution $(1024 \mathrm{x}$ 1024 pixels). Duplicate frames were removed from the raw VLC screen capture videos (the first frame out of every 10 frames is kept, and the following 9 frames of those 10 are discarded). The LCTEM video frames that are displayed in Figures 1,2,3,7 and Figures S4,S6 are single frames extracted from the VLC capture videos. Videos S1-S11 (.qt) were created using Camtasia Studio (TechSmith Corporation, Okemos, MI) video editing software to truncate the raw LCTEM video clips to the desired starting and ending points, and to adjust the video playback speed (see Video captions in SI for video speeds).

Detailed descriptions of the LCTEM video MOTA analysis, quantitative analysis of the MOTA data, ADOMA analysis of the micelles' motion, cryo-TEM sample preparation, and the coarse-grained modeling and simulation approach can be found in the SI (Supporting Methods section).

analysis, ADOMA analysis, coarse-grained modeling and simulation), Supporting Discussion (fusion simulation in Figure 6D and Video S15), Supporting Figures, Supporting Tables, Supporting Video Captions (for Videos S1-S15), and Supporting References. Additionally, videos files (.qt) of all the in situ LCTEM data (Videos S1-S11) and the video files for the four micelle fusion simulations (Videos S12-S15) are included in the SI as separate 
files. The Supporting Information is available free of charge on the ACS Publications website.

\section{AUTHOR INFORMATION}

\section{Corresponding Author}

*Nathan C. Gianneschi

nathan.gianneschi@northwestern.edu

\section{Author Contributions}

The manuscript was written through contributions of all authors. All authors have given approval to the final version of the manuscript.

\section{ACKNOWLEDGMENT}

LRP is supported by the National Institute of Biomedical Imaging and Bioengineering of the National Institutes of Health under award number F32EB021859. JPP is supported by the 4TU HighTech Materials research program 'New Horizons in designer materials.' CP is supported by the National Science Foundation under award number 1334012 and the Air Force Office of Scientific Research under award number FA9550-16-1-0110. Work conducted at UC San Diego and the University of Chicago was supported by a MURI from the Army Research Office under award number W911NF-15-1-0568. We thank the cryo-electron microscopy facility supported by our colleague Prof. Timothy Baker (UC San Diego) funded by the National Institutes of Health. All data reported in this paper are available in the Supporting Information files.

\section{ABBREVIATIONS}

ADOMA, anomalous diffusion object-motion analysis; CMC, critical micelle concentration; fBm, fraction Brownian motion; fGn, fractional Gaussian noise; LCTEM, Liquid-Cell Transmission Electron Microscopy; MOTA, multi-object tracking algorithm; $N_{\text {agg }}$, aggregation number

\section{REFERENCES}

(1) Lehn, J.-M. Science 2002, 295, 2400-2403

(2) Davis, H. T.; Bates, F. S. Macromolecules 2003, 36, 953-955.

(3) Choi, S. H.; Lodge, T. P.; Bates, F. S. Phys. Rev. Lett. 2010, 104, 1-4.

(4) Lu, J.; Bates, F. S.; Lodge, T. P. ACS Macro Lett. 2013, 2, 451455.

(5) Cavicchi, K. A.; Lodge, T. P. J. Polym. Sci. Part B 2002, 41, 715-724.

(6) Meli, L.; Lodge, T. P. Macromolecules 2009, 42, 580-583.

(7) Gummel, J.; Sztucki, M.; Narayanan, T.; Gradzielski, M. Soft Matter 2011, 7, 5731-5738

(8) Jensen, G. V.; Lund, R.; Gummel, J.; Narayanan, T.; Pedersen, J. S. Angew. Chemie Int. Ed. 2014, 53, 11524-11528.

(9) Nicolai, T.; Colombani, O.; Chassenieux, C. Soft Matter 2010 6,3111

(10) Mai, Y.; Eisenberg, A. Chem. Soc. Rev. 2012, 41, 5969

(11) Choucair, A.; Eisenberg, A. Eur. Phys. J. E. Soft Matter 2003, $10,37-44$

(12) Zhang, L.; Eisenberg, A. J. Am. Chem. Soc. 1996, 118, 31683181 .

(13) Adams, D. J.; Topham, P. D. In Supramolecular Chemistry; John Wiley \& Sons, Ltd, 2012.

(14) Lipowsky, R. Nature 1991, 349, 475-481.

(15) Leng, J.; Egelhaaf, S. U.; Cates, M. E. Europhys. Lett. 2002, 59 311-317.

(16) Sevink, G. J. A.; Zvelindovsky, A. V. Mol. Simul. 2007, 33 , $405-415$.

(17) Nagarajan, R. Colloids and Surfaces 1985, 13, 1-17.

(18) Tanford, C. J. Phys. Chem. 1974, 78, 2469-2479.

(19) Cui, H.; Chen, Z.; Zhong, S.; Wooley, K. L.; Pochan, D. J. Science 2007, 317, 647-650.
Matsen, M.; Bates, F. Macromolecules 1996, 29, 7641-7644. Riess, G. Prog. Polym. Sci. 2003, 28, 1107-1170.

Jain, S.; Bates, F. S. Science 2003, 300, 460-464.

Yu, Y. S.; Zhang, L. F.; Eisenberg, A. Macromolecules 1998, 31, 1144-1154.

Jain, S.; Bates, F. S. Macromolecules 2004, 37, 1511-1523.

Li, Z.; Kesselman, E.; Talmon, Y.; Hillmyer, M. A.; Lodge, T. P. Science 2004, 306, 98-101.

Wang, X.; Guerin, G.; Wang, H.; Wang, Y.; Manners, I.; Winnik, M. a. Science 2007, 317, 644-648.

Li, X.; Gao, Y.; Boott, C. E.; Winnik, M. A.; Manners, I. Nat. Commun. 2015, 6, 8127.

Stejskal, J.; Hlavat, D.; Sikora, A.; Kohbk, C.; Ple, J.; Kratochvil, P. Polymer (Guildf). 1992, 33, 3675-3685.

Halperin, A. Macromolecules 2011, 44, 5072-5074.

Kelley, E. G.; Murphy, R. P.; Seppala, J. E.; Smart, T. P.; Hann, S. D.; Sullivan, M. O.; Epps, T. H. Nat. Commun. 2014, 5, 3599. Murphy, R. P.; Kelley, E. G.; Rogers, S. a; Sullivan, M. O.; Epps, T. H. ACS Macro Lett. 2014, 3, 1106-1111.

Dormidontova, E. E. Macromolecules 1999, 32, 7630-7644.

Won, Y. Y.; Brannan, A. K.; Davis, H. T.; Bates, F. S. J. Phys. Chem. B 2002, 106, 3354-3364.

Moughton, A. O.; Hillmyer, M. A.; Lodge, T. P. Macromolecules 2012, 45, 2-19.

Leng, J.; Egelhaaf, S. U.; Cates, M. E. Biophys. J. 2003, 85, 1624-1646.

Nyrkova, I. a.; Semenov, A. N. Macromol. Theory Simulations 2005, 14, 569-585.

Halperin, a; Alexander, S. Macromolecules 1989, 22, 24032412.

Rharbi, Y.; Winnik, M. a. Adv. Colloid Interface Sci. 2001, 89 90, 25-46.

He, X.; Schmid, F. Phys. Rev. Lett. 2008, 100, 137802-137805. Guan, W.; Zhou, W.; Lu, C.; Tang, B. Z. Angew. Chemie Int. Ed. 2015, 54, 15160-15164.

Lund, R.; Willner, L.; Richter, D. In Controlled Polymerization and Polymeric Structures: Flow Microreactor Polymerization, Micelles Kinetics, Polypeptide Ordering, Light Emitting Nanostructures; Abe, A., Lee, K.-S., Leibler, L., Kobayashi, S., Eds.; Springer International Publishing: Cham, 2013; pp 51158.

Zana, R. In Dynamics of Surfactant Self-Assemblies; Surfactant Science; CRC Press, 2005; pp 37-73.

Lu, J.; Bates, F. S.; Lodge, T. P. Macromolecules 2015, 48, 2667-2676.

Lu, J.; Bates, F. S.; Lodge, T. P. Macromolecules 2016, 49, 1405-1413.

Ma, Y.; Lodge, T. P. Macromolecules 2016, 49, 9542-9552.

Kammeyer, J. K.; Blum, A. P.; Adamiak, L.; Hahn, M. E.; Gianneschi, N. C. Polym. Chem. 2013, 4, 3929-3933.

Callmann, C. E.; Barback, C. V.; Thompson, M. P.; Hall, D. J.; Mattrey, R. F.; Gianneschi, N. C. Adv. Mater. 2015, 27, 46114615.

Blum, A. P.; Kammeyer, J. K.; Yin, J.; Crystal, D. T.; Rush, A. M.; Gilson, M. K.; Gianneschi, N. C. J. Am. Chem. Soc. 2014, 136, 15422-15437.

Ungerleider, J. L.; Kammeyer, J. K.; Braden, R. L.; Christman, K. L.; Gianneschi, N. C. Polym. Chem. 2017, 8, 5213-5219.

Blum, A. P.; Kammeyer, J. K.; Gianneschi, N. C. Chem. Sci. 2016, 7, 989-994.

Sanford, M. S.; Love, J. A.; Grubbs, R. H. Organometallics 2001, 20, 5314-5318.

Lund, R.; Willner, L.; Monkenbusch, M.; Panine, P.; Narayanan, T.; Colmenero, J.; Richter, D. Phys. Rev. Lett. 2009, 102, 188301-188304.

Holder, S. J.; Sommerdijk, N. a. J. M. Polym. Chem. 2011, 2, 1018.

McKenzie, B. E.; Holder, S. J.; Sommerdijk, N. a J. M. Curr. Opin. Colloid Interface Sci. 2012, 17, 343-349.

McKenzie, B. E.; Friedrich, H.; Wirix, M. J. M.; de Visser, J. F.; Monaghan, O. R.; Bomans, P. H. H.; Nudelman, F.; Holder, S. J.; Sommerdijk, N. a. J. M. Angew. Chemie Int. Ed. 2015, 54, 2457-2461.

Barnhill, S. A.; Bell, N. C.; Patterson, J. P.; Olds, D. P.; Gianneschi, N. C. Macromolecules 2015, 48, 1152-1161. 
Schneider, N. M.; Norton, M. M.; Mendel, B. J.; Grogan, J. M.; Ross, F. M.; Bau, H. H. J. Phys. Chem. C 2014, 118, 2237322382 .

(58) Abellan, P.; Woehl, T. J.; Parent, L. R.; Browning, N. D.; Evans, J. E.; Arslan, I. Chem. Commun. 2014, 50, 4873-4880. Parent, L. R.; Robinson, D. B.; Woehl, T. J.; Ristenpart, W. D.; Evans, J. E.; Browning, N. D.; Arslan, I. ACS Nano 2012, 6 , 3589-3596.

(60) Patterson, J. P.; Abellan, P.; Denny, M. S.; Park, C.; Browning, N. D.; Cohen, S. M.; Evans, J. E.; Gianneschi, N. C. J. Am. Chem. Soc. 2015, 137, 7322-7328.

(61)

Woehl, T. J.; Jungjohann, K. L.; Evans, J. E.; Arslan, I.; Ristenpart, W. D.; Browning, N. D. Ultramicroscopy 2013, 127, 53-63.

(62) Ross, F. M. Liquid Cell Electron Microscopy, 1st ed.; Cambridge University Press: New York, 2016.

(63) Park, C.; Woehl, T. J.; Evans, J. E.; Browning, N. D. IEEE Trans. Pattern Anal. Mach. Intell. 2015, 37, 611-624.

(64) Vo, G.; Park, C. IEEE Trans. Pattern Anal. Mach. Intell. 2016, $1-13$.

(65) Bakalis, E.; Höfinger, S.; Venturini, A.; Zerbetto, F. J. Chem Phys. 2015, 142, 215102.

(66) Golding, I.; Cox, E. C. Phys. Rev. Lett. 2006, 96, 14-17.

(67) Weber, S. C.; Spakowitz, A. J.; Theriot, J. a. Phys. Rev. Lett. 2010, 104, 27-30.

(68) Jeon, J. H.; Tejedor, V.; Burov, S.; Barkai, E.; Selhuber-Unkel, C.; Berg-S??rensen, K.; Oddershede, L.; Metzler, R. Phys. Rev. Lett. 2011, 106, 2-5.

(69) Tabei, S. M.; Burov, S.; Kim, H. Y.; Kuznetsov, A.; Huynh, T.; Jureller, J.; Philipson, L. H.; Dinner, a R.; Scherer, N. F. Proc Natl Acad Sci U S A 2013, 110, 4911-4916.

(70) Goychuk, I. In Advances in Chemical Physics; John Wiley \& Sons, Inc., 2012; pp 187-253.

(71) Havlin, S.; Ben-Avraham, D. Adv. Phys. 2002, 51, 187-292.

(72) Deng, W.; Barkai, E. Phys. Rev. E - Stat. Nonlinear, Soft Matter Phys. 2009, 79, 1-7.

(73) Jeon, J.-H.; Monne, H. M. S.; Javanainen, M.; Metzler, R. Phys. Rev. Lett. 2012, 109, 188103-188105.

(74) Woehl, T. J.; Prozorov, T. J. Phys. Chem. C 2015, 119, 2126121269.

(75) Woehl, T. J.; Prozorov, T. J. Phys. Chem. C 2015, 119, 2126121269.

(76) Koch, W.; Friedlander, S. K. J. Colloid Interface Sci. 1990, 140, 419-427.

(77) Hawa, T.; Zachariah, M. R. J. Aerosol Sci. 2006, 37, 1-15.

(78) Frenkel, J. J. Phys. 1945, 9, 385-391.

(79) Friedlander, S.; Wu, M. Phys. Rev. B 1994, 49, 3622-3624.

(80) Guevara-Chapa, E.; Mejía-Rosales, S. J. Nanoparticle Res. 2014, 16, 2757-2767.
(81) Langmuir, I. J. Chem. Phys. 1938, 6, 873-896.

(82) Johnson, B. K.; Prud'homme, R. K. Phys. Rev. Lett. 2003, 91, 118302.

(83) Li, S.; Zhang, X.; Dong, W.; Wang, W. Langmuir 2008, 24, 9344-9353.

(84) Herrmann, K. W. J. Colloid Interface Sci. 1966, 22, 352-359.

(85) Tori, K.; Nakagawa, T. Kolloid-Zeitschrift und Zeitschrift fur Polym. 1963, 188, 47-52.

(86) Baptista, M. da S.; Cuccovia, I.; Chaimovich, H.; Politi, M. J.; Reed, W. F. J. Phys. Chem. 1992, 96, 6442-6449.

(87) Drinkel, E.; Souza, F. D.; Fiedler, H. D.; Nome, F. Curr. Opin. Colloid Interface Sci. 2013, 18, 26-34.

(88) Obewele, R. O. Polymer Science and Technology, 1st ed.; CRC Press, 2000

(89) Jenkins, A. D. Polymer Science: A Materials Science Handbook, 2nd ed.; Elsevier, 2013.

(90) Yu, X.; Yu, W.; Yi, B.; Wang, X. Chem. Pap. 2009, 63, 432437.

(91) Nakano, M.; Fukuda, M.; Kudo, T.; Endo, H.; Handa, T. Phys. Rev. Lett. 2007, 98, 238101-238104.

(92) Chien, M.; Thompson, M. P.; Lin, E. C.; Gianneschi, N. C. Chem. Sci. 2012, 3, 2690-2694.

(93) Stevens, M. J.; Hoh, J. H.; Woolf, T. B. Phys. Rev. Lett. 2003, 91, 188102-188104.

(94) Shillcock, J. C.; Lipowsky, R. Nat. Mater. 2005, 4, 225-228.

(95) Ortiz, V.; Nielsen, S. O.; Discher, D. E.; Klein, M. L.; Lipowsky, R.; Shillcock, J. J. Phys. Chem. B 2005, 109, 1770817714.

(96) Li, Z.; Dormidontova, E. E. Macromolecules 2010, 43, 35213531.

(97) Daoulas, K. C.; Marcus, M. In Advances in Polymer Science Polymer Science; 2010; pp 197-233.

(98) Groot, R. D.; Rabone, K. L. Biophys. J. 2001, 81, 725-736.

(99) Grafmuller, A.; Shillcock, J.; Lipowsky, R. Phys. Rev. Lett. 2007, 98, 218101-218104.

(100) Muller, M.; Smirnova, Y. G.; Marelli, G.; Fuhrmans, M.; Shi, A.-C. Phys. Rev. Lett. 2012, 108, 228103-228105.

(101) Kita-Tokarczyk, K.; Junginger, M.; Belegrinou, S.; Taubert, A. Adv. Polym. Sci. 2011, 242, 151-201.

(102) Toomey, R.; Mays, J.; Holley, D. W.; Tirrell, M. Macromolecules 2005, 38, 5137-5143.

(103) Randolph, L. M.; Chien, M.; Gianneschi, N. C. Chem. Sci. 2012, 3, 1363-1380.

(104) Amiel, C.; Sikka, M.; Schneider, J. W.; Tsao, Y.; Tirrell, M.; Mays, J. W. Macromolecules 1995, 28, 3125-3134.

(105) Johner, A.; Joanny, J. F. Macromolecules 1990, 23, 5299-5311.

(106) Choi, S.; Bates, F. S.; Lodge, T. P. Macromolecules 2014, 47, 7978-7986. 


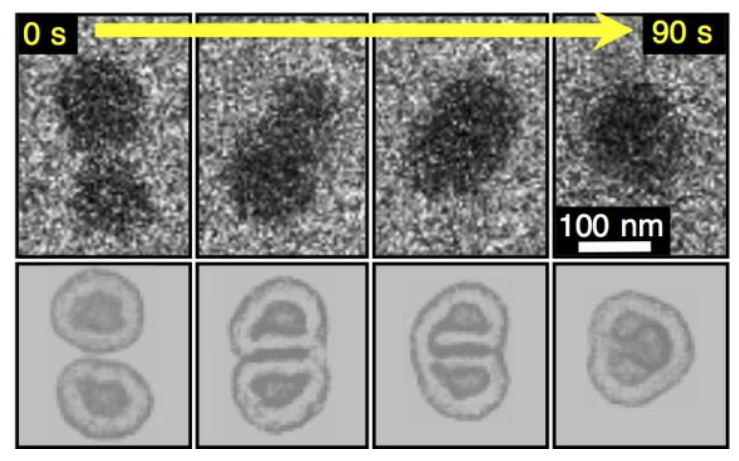

Table of Contents artwork 